\title{
Semiempirical Model for the Ion Impact Ionization of Complex Biological Media
}

\author{
Pablo de Vera, ${ }^{1,2, *}$ Rafael Garcia-Molina, ${ }^{3}$ Isabel Abril, ${ }^{1}$ and Andrey V. Solov'yov, ${ }^{2, \dagger}$ \\ ${ }^{1}$ Departament de Física Aplicada, Universitat d'Alacant, E-03080 Alacant, Spain \\ ${ }^{2}$ Frankfurt Institute for Advanced Studies, Ruth-Moufang-Strasse 1, 60438 Frankfurt am Main, Germany \\ ${ }^{3}$ Departamento de Física-Centro de Investigación en Óptica y Nanofísica, Universidad de Murcia, E-30100 Murcia, Spain
}

(Received 22 September 2012; published 5 April 2013)

\begin{abstract}
We present a semiempirical model for calculating the electron emission from any organic compound after ion impact. With only the input of the density and composition of the target we are able to evaluate its ionization cross sections using plausible approximations. Results for protons impacting in the most representative biological targets (such as water or DNA components) show a very good comparison with experimental data. Because of its simplicity and great predictive effectiveness, the method can be immediately extended to any combination of biological target and charged particle of interest in ion beam cancer therapy.
\end{abstract}

PACS numbers: $87.53 .-\mathrm{j}, 79.20 .-\mathrm{m}, 87.14 . \mathrm{gk}, 87.56 .-\mathrm{v}$

Secondary electron emission is a key step in the mechanism of radiation damage to biomolecular systems induced by ion impact. As a matter of fact, ion beam cancer therapy exploits the particular properties of ion tracks, in which the ionization yield reaches a maximum near the end of their trajectories (the Bragg peak), allowing a precise and narrow energy deposition in deep-seated tumors, minimizing the radiation effects in healthy surrounding tissues [1]. These ejected electrons can produce further ionizations, initiating an avalanche effect, leading to the energy transfer to sensitive biomolecular targets, such as DNA or proteins. But not only is the number of emitted electrons relevant, but also their energy spectrum, since, although high energy electrons are those capable of producing further ionizations, it has been shown that low energy electrons (below ionization threshold) can also produce damage to biomolecules by dissociative electron attachment $[2,3]$.

In order to reach a deeper understanding of ion beam cancer therapy from a fundamental point of view, a great amount of data is needed regarding several and very diverse processes, since the whole mechanism implies steps in very different energy, space, and time scales. Therefore, the problem must be studied within a multiscale approach [4], a fact that motivates an interdisciplinary effort within the European COST Action Nano-IBCT (Nanoscale insights into ion beam cancer therapy) to build a comprehensive database [5]. In this context, ionization data for a wide variety of projectile and organic target combinations, covering a broad range of incident and ejected energies, is needed in order to get insight into micro- and nanometric aspects of radiation damage to biomolecular systems. The aim of this Letter is to present a simple theoretical method that provides the above mentioned required ionization data with the use of little input information, based on the dielectric formalism [6] and some physically motivated approximations. Results are here presented for proton impact, although the methodology can be immediately extended to heavier ions, electrons, and other charged particles.

Although several simple theoretical and semiempirical methods already exist nowadays to calculate the energy spectra of secondary electrons [7] (and are currently in use [8-10]), such as the Rudd formula [11] or the semiclassical binary encounter approximation (BEA) [12], they are limited to some particular targets (atomic ones or small molecules), and its extension to complex biological systems is far from being trivial. Moreover, they can suffer problems resulting from neglecting many-body interactions and target physical state effects. Other more sophisticated approaches can be used instead, like quantum models based on the first Born approximation (or higher order perturbation theory), which can take into account these effects more consistently (e.g., the continuum-distortedwave-eikonal-initial-state method $[13,14])$. Nonetheless, these kind of models are barely applicable for very big and complex biological targets, such as proteins or DNA. On the other hand, the dielectric formalism [6] has been used by several authors to calculate the ionization of condensed biological targets, mainly liquid water $[15,16]$. In the following, we will go beyond this approach, with the use of plausible approximations, applying it to a broader selection of relevant biological targets.

The dielectric formalism [6] introduces the electronic excitation spectrum of the target in the cross section calculation through its energy-loss function (ELF), $\operatorname{Im}[-1 / \epsilon(k, E)]$, where $\epsilon(k, E)$ is the complex dielectric function, with $\hbar k$ and $E$ being, respectively, the momentum and energy transfers in the electronic excitation. Provided that the ELF is experimentally known, many-body interactions and target physical state effects are naturally included in the subsequent calculations. The macroscopic (nonrelativistic) single differential cross section (SDCS) for ionization of the electronic $i$ shell by a projectile of kinetic energy $T$ is [17] 
$\left.\frac{d \Lambda(T, W)}{d W}\right|_{i}=\frac{e^{2}}{\pi \hbar^{2}} \frac{M_{1} Z_{1}^{2}}{T} \int_{k_{-}}^{k_{+}} \frac{d k}{k} \operatorname{Im}\left[\frac{-1}{\epsilon\left(k, B_{i}+W\right)}\right]_{i}$

where we have used $E=B_{i}+W$, with $B_{i}$ and $W$ being, respectively, the binding energy of the $i$ shell and the kinetic energy of the ejected electron. $M_{1}$ and $Z_{1}$ are the mass and charge of the projectile, whereas $\operatorname{Im}\left[-1 / \epsilon\left(k, B_{i}+W\right)\right]_{i}$ is the contribution to the ELF from the $i$-shell ionization. The integration limits, imposed by conservation laws, are $k_{ \pm}=\sqrt{2 M_{1}}(\sqrt{T} \pm \sqrt{T-E})$. The macroscopic cross section is related to the microscopic one, $\sigma$, through $\Lambda=\mathcal{N} \sigma$, where $\mathcal{N}$ is the molecular density of the target. Note that Eq. (1) can be used for different charged projectiles by properly taking into account their charge state, or for electrons by introducing an exchange term in the integrand and imposing the correct integration limits.

According to Eq. (1), we need the contribution of each electronic shell of the target to its ELF in order to calculate its ionization SDCS. The ELF of the target is usually measured for all the excitations and ionizations of the electronic system in the optical limit $(k=0)$. Therefore, we need (i) the experimental optical ELF of the target, (ii) an extension algorithm for $k \neq 0$, and (iii) a method to split the resulting ELF in different electronic shells.

Regarding issue (i), the ELF of a large number of materials has been already measured. Nonetheless, few of them are biological targets. Recently, Tan et al. [18] noticed that the optical-ELF of 13 bioorganic condensed compounds and liquid water are rather similar, with an intense peak around $20-25 \mathrm{eV}$, a fact which led to a parametrization of the optical ELF with a single-Drude function

$$
\operatorname{Im}\left[\frac{-1}{\epsilon(k=0, E)}\right]=\frac{a\left(Z_{2}\right) E}{\left[E^{2}-b\left(Z_{2}\right)^{2}\right]^{2}+c\left(Z_{2}\right)^{2} E^{2}} .
$$

In this equation, $a\left(Z_{2}\right)$ (in $\left.\mathrm{eV}^{3}\right), b\left(Z_{2}\right)$ (in $\mathrm{eV}$ ), and $c\left(Z_{2}\right)$ (in $\mathrm{eV}$ ) represent the height, position, and width of the single-Drude ELF. Whereas, $b\left(Z_{2}\right)$ and $c\left(Z_{2}\right)$ are parametrized as a function of the mean atomic number of the target, $Z_{2}$ [18], $a\left(Z_{2}\right)$ is simply obtained by imposing the accomplishment of the $f$-sum rule [19], linked to the number of electrons in the target, $Z_{2}$, also accounting for the contribution from the inner shells, as explained in Ref. [18]. Using this approach the ELF of an arbitrary bioorganic compound can be estimated, even in the case where no experimental data exist. In Fig. 1 we compare the experimentally determined optical ELF of four condensed organic compounds [20-23] with the results obtained through Eq. (2). As can be seen, the agreement is quite good for all the organic targets, but with larger discrepancies in the case of water. In what follows we will analyze the level of accuracy this parametrization approach yields in the calculation of ionization SDCS.

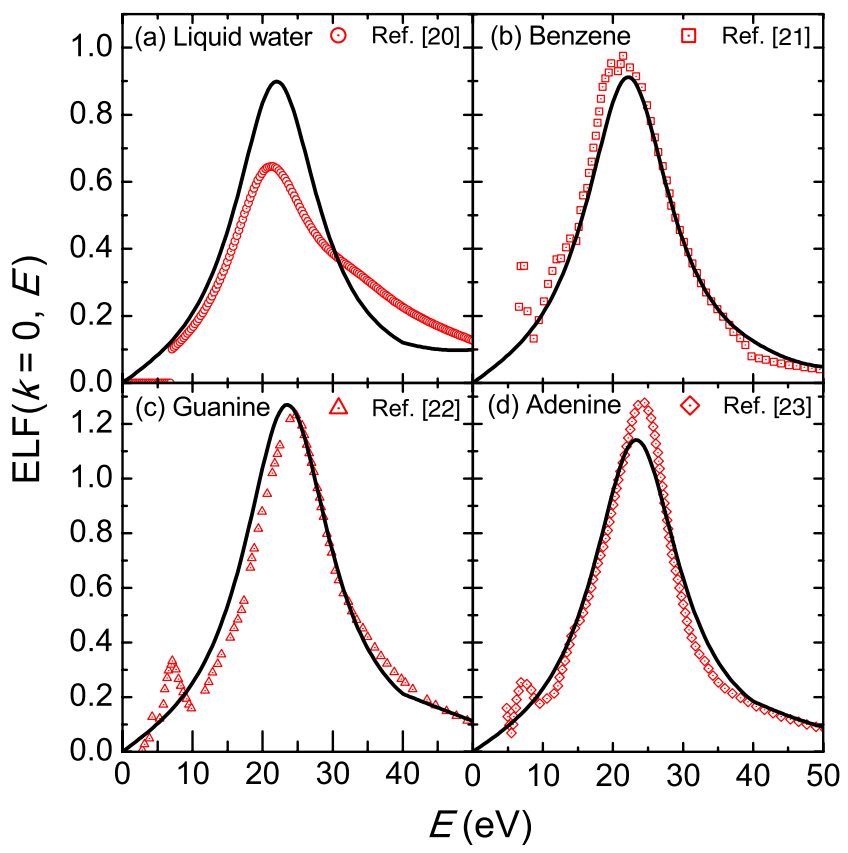

FIG. 1 (color online). Comparison of the optical ELF obtained through the parametric model of Eq. (2) (solid lines) with available experimental data (symbols) for (a) liquid water [20], (b) solid benzene [21], (c) solid guanine [22], and (d) solid adenine [23].

Problem (ii) has been studied for many years, and a wide variety of extension algorithms to extrapolate the optical-ELF to $k \neq 0$ are available [24]. For the sake of simplicity, in this work we use the simple quadratic dispersion relation introduced by Ritchie and Howie [25], with its parameters for liquid water [24], since the model is easier to implement in this way and it gives results of the same level of accuracy as other approximations used in our methodology.

The requirement (iii), i.e., the splitting of the ELF in contributions from different shells, is not a trivial task. This issue has already been addressed for liquid water [15,16], providing parametrizations of the ELF split in ionization and excitation arising from different shells. Nonetheless, to extend this approach to other biological media, especially to large macromolecules, is rather difficult, since the number of electronic levels is much larger than for water. Therefore, here we will apply a specially designed approximation, to be discussed later on. In general, it is possible to obtain information on the mean kinetic and binding energies of the molecular electronic levels by quantum chemistry calculations. These data have been recently obtained in the context of binary encounter calculations for some biological molecules, such as the DNA bases and the sugar-phosphate backbone [8] and some amino acids [9]; this information can be found for other molecules in a National Institute of Standards and Technology database [26]. In order to describe the outer-shell ionization of these molecules, we calculate a mean value of their binding 
energies, $\bar{B}$, and consider that the outer-shell electrons will be ionized if the transferred energy satisfies $E>\bar{B}$. Then, the ejected electron energy is $W=E-\bar{B}$.

The use of such a crude approximation is, nonetheless, justified by the following arguments: first of all, if one looks at the shape of the ELF of liquid water split in excitations and ionizations by the existing parametrizations $[15,16]$, it is clear that our average binding energy will consider as ionizations some excitations above $\bar{B}$, and will neglect some ionizations produced below $\bar{B}$, but most ionizations will be produced at $E>\bar{B}$, and a small number of excitations will occur above this threshold. Moreover, the differences between the real ionization energies of the molecule and the mean binding energy will be of the order of a few electronvolts. Such differences can be neglected when analyzing the transport of the produced secondary electrons. Below we demonstrate that this approximation, in spite of its simplicity, results in a very good comparison with experimental data.

We have applied the aforementioned methodology to calculate the SDCS for proton impact in water, adenine, and benzene, where the binding energies of their electronic levels are $\bar{B}_{\text {water }}=18.13$ [15], $\bar{B}_{\text {adenine }}=20.44$ [8], and $\bar{B}_{\text {benzene }}=17.47 \mathrm{eV}$ [26]. The calculated SDCS are shown in Fig. 2. Since we do not know about experimental data in the condensed phase with which we can compare our results, we have taken the available experimental data in the gas phase [27-30]. We find an overall good agreement, except for small differences at low energies, which can be mostly attributed to phase effects and to the extreme difficulty to precisely determine experimentally these low energies.

In Fig. 2 we show by solid (dashed) lines the SDCS calculated with the experimental (parametrized) optical ELF. Comparison of the results using the experimental and parametrized ELF allows us to estimate the uncertainty coming from the predicted ELF for targets for which there are not experimental data. The maximum differences in the SDCS calculated with the parametrized and the experimental optical ELF are of the order of $30 \%$ at the maximum of the curves in the case of liquid water, which corresponds with the worst ELF prediction shown here. This is a reasonable range of error, taking into account that we can predict, in a simple and universal way, the SDCS for any organic compound from little input information. In general, it is better to use the experimental ELF when it is available. Then, the accuracy and reliability of the method increases significantly. One can see in Figs. 2(a)-2(c) that the experimental ELFs of liquid water, adenine, and benzene give better SDCS than the parameterized ELFs. Therefore, we show in Figs. 2(d)-2(f) the SDCS obtained from the experimental ELFs at several proton energies.

We also compare our calculations and the experiments with the results of other models. In Figs. 2(a)-2(c) we depict by dotted lines the SDCS calculated within the BEA [12], and by a dotted-dashed line the results of the

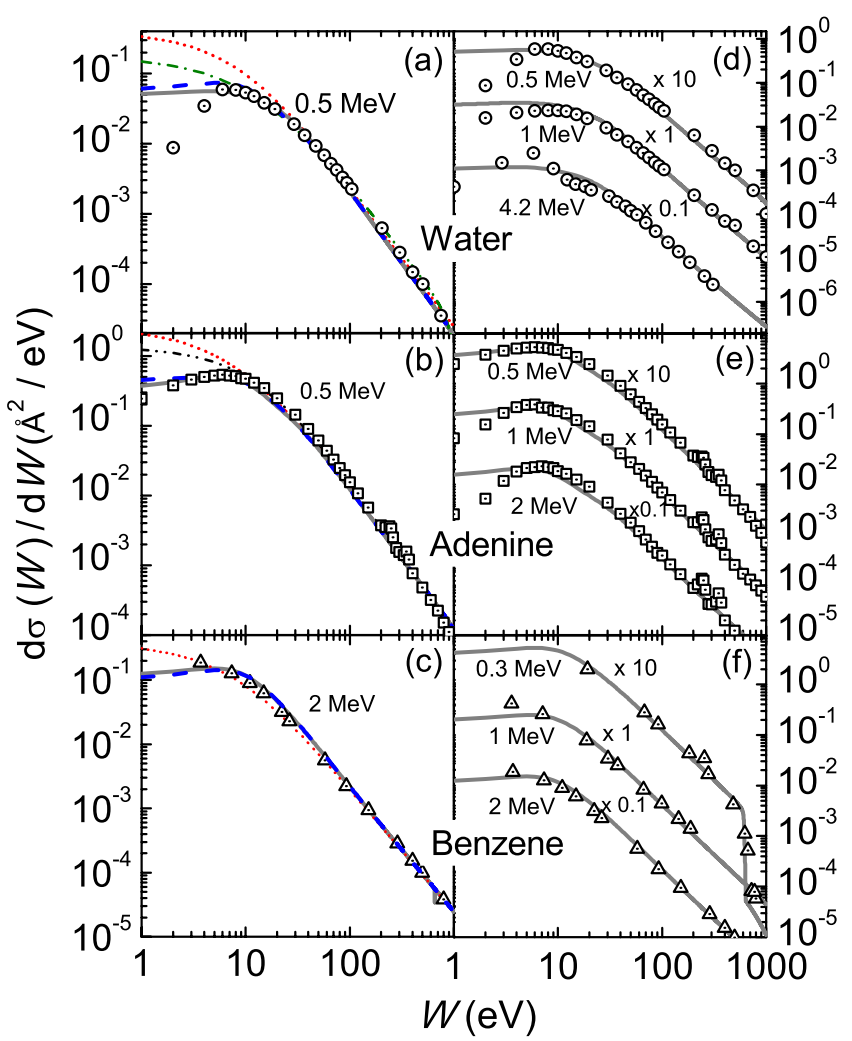

FIG. 2 (color online). Single differential cross sections (SDCSs) for ionization of (a) water, (b) adenine, and (c) benzene by protons of a given energy, obtained from various models; (d), (e), and (f) are the same for several proton energies, obtained with the present model. Symbols represent experimental data: circle with dot [28], square with dot [29,30], triangle with dot [27]; solid lines are our calculations within the dielectric formalism using the experimental ELF of the target, while dashed lines correspond to the parameterized ELF, as described in the text. Dotted lines are calculations performed within the BEA model, while the dash-dotted line is the result of the Rudd formula for water vapor. The dashed-dot-dotted line is an $a b$ initio calculation for the adenine molecule from Ref. [14].

Rudd formula [11] for water vapor. As can be seen, these models also give, in general, good results, but their discrepancies with the experiments at very low ejection energies, say below 10-20 eV, are larger than in our calculations. In Fig. 2(b) we also show an ab initio calculation performed by Galassi et al. [14] (dashed-dot-dotted line), which shows the same kind of discrepancy at low ejection energies. A possible explanation for these differences is that we are considering a more realistic electronic excitation spectrum of the target through its ELF and, because of this, we find smaller differences in comparison with the experimental data. It must be kept in mind that, although these low energy electrons will not travel too far from the point of ejection and they do not have enough energy to produce further ionizations, their description is very important, since they can damage biological molecules by dissociative electron attachment $[2,3]$. 
Once we know the SDCS, we can also calculate from it other key radiobiological quantities of interest such as, for example, the total ionization cross section (TICS)

$$
\Lambda(T)=\int_{0}^{\infty} \frac{d \Lambda(T, W)}{d W} d W
$$

We show in Fig. 3(a) the macroscopic TICS (i.e., inverse mean free path) calculated for proton impact in five representative biological materials relevant for cancer therapy: liquid water, dry DNA $\left(\mathrm{C}_{20} \mathrm{H}_{27} \mathrm{~N}_{7} \mathrm{O}_{13} \mathrm{P}_{2}\right)$, protein, lipid, and the cell nucleus. Their atomic compositions and densities can be found in the ICRU Report 46 [31] and other sources, and a reasonable value of their mean binding energies can be estimated from the values of their molecular components, such as the water molecule, DNA bases and backbone, and amino acids $[8,9,15]$. We also show experimental data for water vapor [28,32,33], which nicely agree with our calculations above $100 \mathrm{keV}$, where the first Born approximation is applicable without further corrections. From our results, it seems that all the biological targets different from water have a larger ionization probability than water. We can also see that the TICS of cell nucleus is only slightly larger than that of liquid water, and that the protein has a slightly larger TICS than the rest of biomaterials.

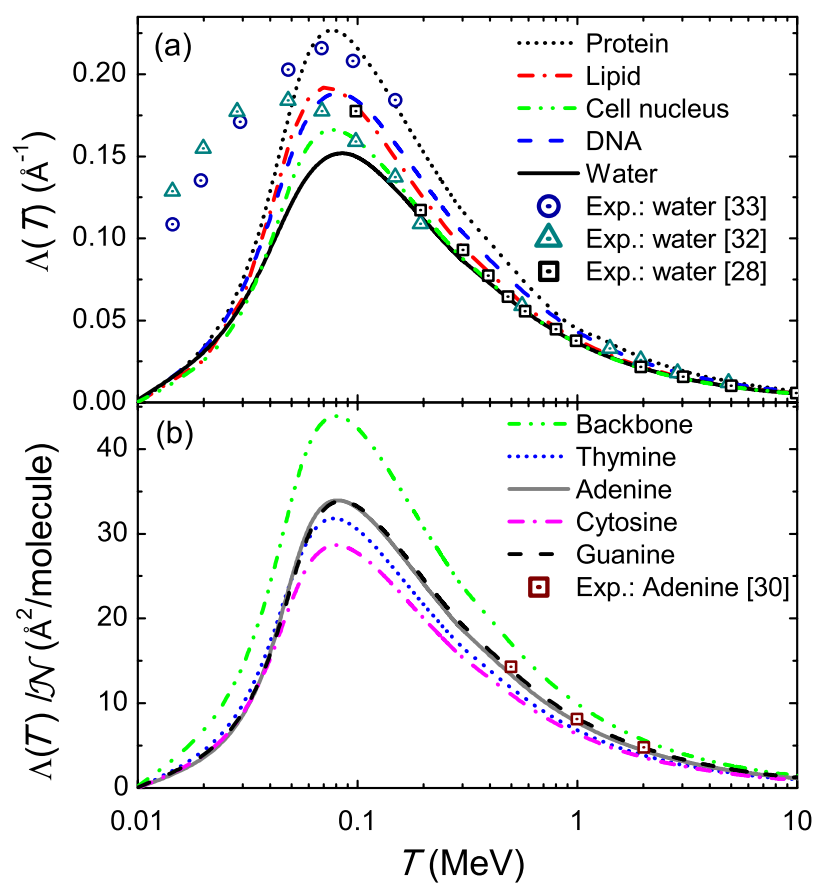

FIG. 3 (color online). (a) Calculated macroscopic TICS for proton impact in liquid water, DNA, protein, lipid, and cell nucleus. (b) Calculated microscopic TICS for proton impact in the DNA components adenine, cytosine, guanine, thymine, and sugar-phosphate backbone. Symbols represent experimental data.
Finally, we show in Fig. 3(b) the microscopic TICS per molecule for proton impact in the DNA molecular components: adenine, cytosine, guanine, thymine, and sugar-phosphate backbone. Their atomic composition can be easily found in the literature, and we have estimated their mean binding energies from quantum chemistry calculations [8,9]. Also shown are experimental data at high energies for adenine [30], which are in excellent agreement with our predictions. Experimental information at low and intermediate energies is scarce [34] and seems too large in comparison with most of the experimental and calculated data $[14,35]$. We do not plot these data in Fig. 3(b) due to the large scale differences. Our method allows us to estimate the ionization probability of each constituent of the DNA molecule, which gives important information on the sensitivity of each one to radiation damage. According to these results, the DNA backbone is the most probable part of the DNA to be ionized by proton impact (a similar behavior was previously observed for electron impact in Ref. [8]; also, recent theoretical estimates [36] point towards sugar-phosphate $\mathrm{C}-\mathrm{O}$ bond cleavage due to interaction with low energy electrons) and, between bases, adenine and guanine are the most sensitive to protonimpact ionization. This fact could have important implications in the DNA damage, since it seems that single or double strand breaks could be more probable than base damage, or that regions of the DNA with a bigger concentration of adenine or guanine would be more likely damaged by radiation than other parts of the genome, attending to direct ionization effects. Nonetheless, it must be kept in mind that other processes, such as the interactions of the ejected electrons, the attack of free radicals, the evolution of the molecule after ionization, or dissociative electron attachment, play an important role in the description of radiation damage too.

Much more information can be obtained with the proposed method, like the number of emitted electrons, the average energy of electrons, SDCS and TICS for other biological targets and projectiles, etc., but these tasks will be addressed in future studies. Here, we have presented the essentials of the model which, using little input information and physically motivated approximations, can provide very useful information about the ion impact ionization of a huge number of relevant biological targets, for which data are lacking, both experimentally and theoretically, and for which there is a need for insight into ionization processes. The model makes use of an empirical parametrization of the optical ELF of organic compounds, which gives quite good results, together with the dielectric formalism to take into account the many-body and physical state effects of the target, improving the results of SDCS obtained by other models at low ejection energies. We also want to stress that our model can be easily extended to ions heavier than protons, in different charge states, as well as to electron impact ionization, by introducing appropriate 
corrections, such as the description of the electronic structure of the ion, or exchange and relativistic (if needed) corrections for electrons. Refinements and improvements to this model will be introduced in future works, especially regarding the empirical parametrization of the ELF and the choice of the mean binding energy (which, of course, can also be achieved by the best fitting of the model to existing experimental data), but, from the current results, it is clear that this simple model has a great predictive effectiveness, with the need of few input data about the complex biological target.

The authors want to thank for the fruitful discussion and helpful data provided by Dr. E. Surdutovich, Dr. E. Scifoni, Dr. D. Emfietzoglou, and Dr. C. Champion. We recognize the financial support from the Spanish Ministerio de Economía y Competitividad and the European Regional Development Fund within the Project No. FIS2010-17225, and from the Conselleria d'Educació, Cultura i Esport de la Generalitat Valenciana for granting PdV within the VALi+d program. Support from the COST Action MP1002 Nano-IBCT is gratefully acknowledged.

*Corresponding author. pablo.vera@ua.es

On leave from A.F. Ioffe Physical-Technical Institute, Politechnicheskaya 26, 194021 St. Petersburg, Russia.

[1] D. Schardt, T. Elsässer, and D. Schulz-Ertner, Rev. Mod. Phys. 82, 383 (2010).

[2] B. Boudaïffa, P. Cloutier, D. Hunting, M. A. Huels, and L. Sanche, Science 287, 1658 (2000).

[3] X. Pan, P. Cloutier, D. Hunting, and L. Sanche, Phys. Rev. Lett. 90, 208102 (2003).

[4] A. V. Solov'yov, E. Surdutovich, E. Scifoni, I. Mishustin, and W. Greiner, Phys. Rev. E 79, 011909 (2009).

[5] Workshop on Nano-IBCT data base development, Vienna, Austria, 2012. Book of abstracts available online at: http://fias.uni-frankfurt.de/fileadmin/fias/nano-ibct/ NanoIBCTbooklet.pdf.

[6] J. Lindhard, K. Dan. Vidensk. Selsk. Mat. Fys. Medd. 28, 8 (1954).

[7] M. E. Rudd, Y.-K. Kim, T. Märk, J. Schou, N. Stolterfoht, and L.H. Toburen, Secondary Electron Spectra from Charged Particle Interactions (International Commission on Radiation Units and Measurements, Bethesda, MD, 1996), (ICRU 55).

[8] Ph. Bernhardt and H. G. Paretzke, Int. J. Mass Spectrom. 223-224, 599 (2003).

[9] A. Peudon, S. Edel, and M. Terrisol, Radiat. Prot. Dosim. 122, 128 (2006).

[10] E. Scifoni, E. Surdutovich, and A. V. Solov'yov, Phys. Rev. E 81, 021903 (2010).

[11] M. E. Rudd, Y.-K. Kim, D. H. Madison, and T. J. Gay, Rev. Mod. Phys. 64, 441 (1992).
[12] L. Vriens, Proc. Phys. Soc. London 90, 935 (1967).

[13] D. S. F. Crothers and J.F. McCann, J. Phys. B 16, 3229 (1983).

[14] M. E. Galassi, C. Champion, P. F. Weck, R. D. Rivarola, O. Fojón, and J. Hanssen, Phys. Med. Biol. 57, 2081 (2012).

[15] M. Dingfelder, D. Hantke, M. Inokuti, and H. G. Paretzke, Radiat. Phys. Chem. 53, 1 (1999).

[16] D. Emfietzoglou, Radiat. Phys. Chem. 66, 373 (2003).

[17] D. Emfietzoglou, Interaction of Radiation with Matter, edited by H. Nikjoo, S. Uehara, and D. Emfietzoglou (CRC, Boca Raton, 2012), Sec. III.

[18] Z. Tan, Y. Xia, M. Zhao, X. Liu, F. Li, B. Huang, and Y. Ji, Nucl. Instrum. Methods Phys. Res., Sect. B 222, 27 (2004).

[19] M. Altarelli and D. Y. Smith, Phys. Rev. B 9, 1290 (1974).

[20] H. Hayashi, N. Watanabe, Y. Udagawa, and C. C. Kao, Proc. Natl. Acad. Sci. U.S.A. 97, 6264 (2000).

[21] J. A. LaVerne and S. M. Pimblott, J. Phys. Chem. 99, 10540 (1995).

[22] L. C. Emerson, M. W. Williams, I'lan Tang, R. N. Hamm, and E. T. Arakawa, Radiat. Res. 63, 235 (1975).

[23] E. T. Arakawa, L. C. Emerson, S. I. Juan, J. C. Ashley, and M. W. Williams, Photochem. Photobiol. 44, 349 (1986).

[24] R. Garcia-Molina, I. Abril, I. Kyriakou, and D. Emfietzoglou, Radiation Damage in Biomolecular Systems, edited by G. García Gómez-Tejedor and M. C. Fuss (Springer, Dordrecht, 2012), Chap. 15.

[25] R. H. Ritchie and A. Howie, Philos. Mag. 36, 463 (1977).

[26] Y.-K. Kim et al., Electron-Impact Ionization Cross Section for Ionization and Excitation Database (version 3.0) (National Institute of Standards and Technology, Gaithersburg, MD, 2004), http://www.nist.gov/pml/data/ ionization/index.cfm.

[27] W.E. Wilson and L.H. Toburen, Phys. Rev. A 11, 1303 (1975).

[28] W.E. Wilson, J.H. Miller, L.H. Toburen, and S.T. Manson, J. Chem. Phys. 80, 5631 (1984).

[29] Y. Iriki, Y. Kikuchi, M. Imai, and A. Itoh, Phys. Rev. A 84, 032704 (2011).

[30] Y. Iriki, Y. Kikuchi, M. Imai, and A. Itoh, Phys. Rev. A 84, 052719 (2011).

[31] D. R. White, R. V. Griffith, and I. J. Wilson, Photon, Electron, Proton and Neutron Interaction Data for Body Tissues (International Commission on Radiation Units and Measurements, Bethesda, MD, 1992), (ICRU 46).

[32] M. E. Rudd, T. V. Goffe, R. D. DuBois, and L. H. Toburen, Phys. Rev. A 31, 492 (1985).

[33] M. A. Bolorizadeh and M. E. Rudd, Phys. Rev. A 33, 888 (1986).

[34] J. Tabet, S. Eden, S. Feil, H. Abdoul-Carime, B. Farizon, M. Farizon, S. Ouaskit, and T. D. Märk, Phys. Rev. A 82, 022703 (2010).

[35] C. Champion, P.F. Weck, H. Lekadir, M.E. Galassi, O. A. Fojón, P. Abufager, R. D. Rivarola, and J. Hanssen, Phys. Med. Biol. 57, 3039 (2012).

[36] J. Simons, Adv. Quantum Chem. 52, 171 (2007). 